\title{
Subspecialty certification in thoracic oncology: Toward your next diploma
}

\author{
Shawn S. Groth, MD, and Bryan M. Burt, MD
}

\author{
From the Division of General Thoracic Surgery, Department of Surgery, Baylor College of Medicine, Houston, \\ Tex \\ Disclosures: Authors have nothing to disclose with regard to commercial support. \\ Received for publication Aug 4, 2017; accepted for publication Aug 7, 2017. \\ Address for reprints: Bryan M. Burt, MD, Division of General Thoracic Surgery, Department of Surgery, Baylor \\ College of Medicine, One Baylor Plaza, BCM 390, Houston, TX 77005 (E-mail: bryan.burt@bcm.edu). \\ J Thorac Cardiovasc Surg 2017;154:1393-4 \\ $0022-5223 / \$ 36.00$ \\ Copyright (C) 2017 by The American Association for Thoracic Surgery \\ http://dx.doi.org/10.1016/j.jtcvs.2017.08.001
}

\section{Expertise is the mantra of modern medicine.}

\section{-Atul Gawande, \\ The Checklist Manifesto: \\ How to Get Things Right}

The current health care environment in the United States, embodying constraint on costs and leaning on accountable care models of health care delivery, has placed increasing emphasis on the delivery of high-quality patient care. Such demands have been replied to with mounting subspecialization of medical practices, teamwork, and care coordination; the essence of multidisciplinary patient care. The subspecialty field of thoracic oncology represents an intersection of thoracic surgery, medical oncology, and radiation oncology, and in no other field are these principles more important.

In this issue of the Journal is a report produced from a collaboration of European and American thoracic surgical oncologists outlining the rationale and the current and future directions of competency training, and potential certification, in the subspecialty of "thoracic oncology.", This timely report reflects on our current era, in which we have seen rapid parallel advances in thoracic surgery (including the precipitous adoption of minimally invasive surgical techniques), medical oncology (including unprecedented responses to immunotherapy and targeted therapy), and radiation oncology (including the rise of sophisticated ablative radiation modalities). The management of thoracic malignancies has therefore become increasingly complex. This complexity underscores the central role for multidisciplinary review, so much so in fact that a reasonable case can be made for multidisciplinary review of all new thoracic oncology diagnoses, including early stage disease.

In their article in this issue, Massard and colleagues ${ }^{2}$ provide a cogent argument to provide formal training and certification in thoracic oncology. At the center of this training and certification are the quintessential multidisciplinary patient care conferences, so often referred to as "tumor boards." Massard and colleagues ${ }^{2}$ suggest that such a certificate may one day be required for a leadership role in these and maintenance.

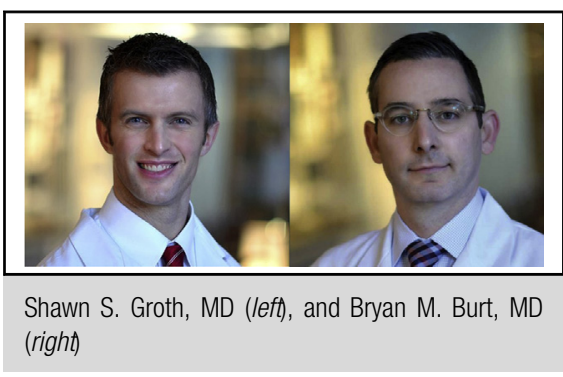

Central Message

A subspecialty diploma in thoracic oncology is on our horizon.

See Article page 1387.

meetings, and they also suggest that the thoracic surgeon should take the front seat in such a role. Further, these conferences are rich in experience and education and can be predicted to be an integral force in certificate achievement

There will undoubtedly be challenges to the mechanics and implementation of a certificate in thoracic oncology. Whereas elements of certificate training for the thoracic surgeon will likely be crystallized in the thoracic surgical training curriculum, it is unclear how the practicing thoracic surgeon would attain certification. There will be further challenges in providing incentives for surgeons and health care institutions to embrace the importance of certification, and there will be credentialing questions for hospitals and cancer centers. Nevertheless, the benefits of thoracic oncology specialization to our patients has become glaringly obvious and will remain the driving force for this movement. Lung cancer is the second most common cancer and the number one cause of cancer-related deaths in the world, and there are now innumerable reports, as well referenced by Massard and colleagues, ${ }^{2}$ demonstrating that multidisciplinary approaches to the treatment of patients with lung cancer results have improved patient outcomes and quality of life.

In closing, we can expect to see continued forward progress on this thoracic oncology certification initiative, and the article of Massard and colleagues ${ }^{2}$ provides important multinational perspectives on the current state of this evolving subspecialty. From this article, our readers will likely perceive a somewhat more advanced position of our European colleagues in this arena, particularly with regard to the Harmonized Education in Respiratory Medicine for European Specialist (HERMES) initiatives in education 
and certification that will extend to thoracic oncology. We should view these initiatives, and the article of Massard and colleagues ${ }^{2}$ itself, as a roadmap for steps forward on this side of the Atlantic, and as an open door for international collaboration.

\section{References}

1. Gawande A. The checklist manifesto: how to get things right. New York: Metropolitan Books; 2009.

2. Massard G, Antonoff MB, Noel J-L, Brunelli A, Farjah F, Lanuti M, Van Raemdonck D, et al. Transatlantic editorial: Thoracic surgeons need recognition of competence in thoracic oncology. J Thorac Cardiovasc Surg. 2017;154:1387-92. 